Supplement of Atmos. Chem. Phys. Discuss., 14, 26463-26494, 2014

http://www.atmos-chem-phys-discuss.net/14/26463/2014/

doi:10.5194/acpd-14-26463-2014-supplement

(C) Author(s) 2014. CC Attribution 3.0 License.

(c) (1)

\title{
Frequency of new particle formation events in the urban Mediterranean
} climate

M. Brines et al.

Correspondence to: M. Brines (mariola.brines@idaea.csic.es) 
Table S1: Exact locations of the selected SMPS sampling sites, their elevation and site type.

\begin{tabular}{|c|c|c|c|c|c|}
\hline City & Site & Latitude & Longitude & $\begin{array}{c}\text { Elevatio } \\
\mathrm{n} \\
\text { (m.a.s.l. } \\
\text { ) }\end{array}$ & Site type \\
\hline $\begin{array}{l}\text { Barcelona, } \\
\text { Spain }\end{array}$ & $\begin{array}{l}\text { Palau } \\
\text { Reial }\end{array}$ & $\begin{array}{c}41^{\circ} 23^{\prime} 14^{\prime \prime} \\
\mathrm{N}\end{array}$ & $2^{\circ} 6^{\prime} 56^{\prime \prime} E$ & $78 \mathrm{~m}$ & $\begin{array}{c}\text { Urban } \\
\text { backgroun } \\
d\end{array}$ \\
\hline Madrid, Spain & CIEMAT & $\begin{array}{c}40^{\circ} 27^{\prime} 30^{\prime \prime} \\
\mathrm{N}\end{array}$ & $3^{\circ} 43^{\prime} 30 " \mathrm{~W}$ & $655 \mathrm{~m}$ & $\begin{array}{c}\text { Suburban } \\
\text { backgroun } \\
d\end{array}$ \\
\hline Rome, Italy & $\begin{array}{c}\text { Montelibret } \\
\mathrm{ti}\end{array}$ & $\begin{array}{c}42^{\circ} 06^{\prime} 38^{\prime \prime} \\
N\end{array}$ & $12^{\circ} 38^{\prime} 05^{\prime \prime E}$ & $47 \mathrm{~m}$ & $\begin{array}{l}\text { Regional } \\
\text { backgroun } \\
d\end{array}$ \\
\hline $\begin{array}{l}\text { Brisbane, } \\
\text { Australia }\end{array}$ & QUT & $\begin{array}{c}27^{\circ} 28^{\prime} 43^{\prime \prime} \\
S\end{array}$ & $153^{\circ} 1^{\prime} 44^{\prime \prime} \mathrm{E}$ & $10 \mathrm{~m}$ & $\begin{array}{c}\text { Urban } \\
\text { backgroun } \\
\mathrm{d}\end{array}$ \\
\hline $\begin{array}{c}\text { Los Angeles, } \\
\text { USA }\end{array}$ & USC & $34^{\circ} 1^{\prime} 9^{\prime \prime N}$ & $\begin{array}{c}118^{\circ} 16^{\prime} 39^{\prime \prime} \\
W\end{array}$ & $61 \mathrm{~m}$ & $\begin{array}{c}\text { Urban } \\
\text { backgroun } \\
\mathrm{d}\end{array}$ \\
\hline
\end{tabular}

Table S2: Sampling period of the SMPS instruments and their characteristics and size range at each selected city.

\begin{tabular}{|c|c|c|c|c|}
\hline City & Sampling period & SMPS model & $\begin{array}{l}\text { SMPS size } \\
\text { range }\end{array}$ & Size bins \\
\hline $\begin{array}{c}\text { Barcelona } \\
\text { (BCN) }\end{array}$ & $\begin{array}{c}30 / 07 / 2012- \\
04 / 08 / 2013 \\
(7295 \mathrm{~h})\end{array}$ & $\begin{array}{c}\text { TSI (DMA } \\
3081, \text { CPC } \\
3772)\end{array}$ & $\begin{array}{c}11.3-358.7 \\
\mathrm{~nm}\end{array}$ & 97 \\
\hline $\begin{array}{l}\text { Madrid } \\
\text { (MAD) }\end{array}$ & $\begin{array}{c}10 / 01 / 2007- \\
12 / 12 / 2008 \\
(12482 \mathrm{~h})\end{array}$ & $\begin{array}{c}\text { TSI (DMA } \\
3071 \text {, CPC } \\
3022)\end{array}$ & $\begin{array}{c}17.5-572.9 \\
n m\end{array}$ & 34 \\
\hline $\begin{array}{l}\text { Rome } \\
\text { (ROM) }\end{array}$ & $\begin{array}{c}26 / 09 / 2007- \\
07 / 05 / 2009 \\
(3373 \mathrm{~h})\end{array}$ & $\begin{array}{c}\text { TSI (DMA } \\
3081, \text { CPC } \\
3775)\end{array}$ & $\begin{array}{c}15.1-224.7 \\
\mathrm{~nm} \\
(10.2-615.3 \\
\mathrm{nm})\end{array}$ & $\begin{array}{c}76 \\
(87,93, \\
104)\end{array}$ \\
\hline $\begin{array}{c}\text { Brisbane } \\
\text { (BNE) }\end{array}$ & $\begin{array}{c}01 / 01 / 2009- \\
31 / 12 / 2009 \\
(6227 \mathrm{~h})\end{array}$ & $\begin{array}{c}\text { TSI (EC } \\
3080, \text { CPC } \\
3781)\end{array}$ & $\begin{array}{c}10.2-101.8 \\
\mathrm{~nm}\end{array}$ & 65 \\
\hline $\begin{array}{c}\text { Los } \\
\text { Angeles } \\
\text { (LA) }\end{array}$ & $\begin{array}{c}04 / 09 / 2009- \\
10 / 12 / 2009 \\
(2184 \mathrm{~h})\end{array}$ & $\begin{array}{c}\text { TSI (DMA } \\
3081, \text { CPC } \\
3022 A) \\
\end{array}$ & $\begin{array}{c}15.7-371.8 \\
n m\end{array}$ & 89 \\
\hline
\end{tabular}


Table S3: Summary of the measurements and sampling period at the selected cities. $\mathrm{V}^{+}$indicates the measurement site is different to the SMPS site.

\begin{tabular}{|c|c|c|c|c|c|c|c|c|c|c|c|c|c|c|c|c|c|}
\hline \multirow[b]{2}{*}{ City } & \multirow[b]{2}{*}{ Ancillary data site } & \multicolumn{10}{|c|}{ Meteorological data Gaseous Pollutants } & \multicolumn{4}{|c|}{ Particulate Matter } & \multicolumn{2}{|c|}{ Other } \\
\hline & & $\mathrm{T}$ & $\mathrm{RH}$ & $\begin{array}{l}\text { ws/ } \\
\text { wd }\end{array}$ & Rain & SR & $\mathrm{NO}$ & $\mathrm{NO}_{2}$ & $\mathrm{O}_{3}$ & $\mathrm{CC}$ & $\mathrm{SO}_{2}$ & $\mathrm{PM}_{10}$ & $\mathrm{PM}_{2.5}$ & $\mathrm{PM}_{1}$ & $\mathrm{NO}_{3}$ & $\mathrm{~N}$ & $\mathrm{BC}$ \\
\hline Barcelona (BCN) & Palau Reial, Fac. of Physics ${ }^{+}$ & $\mathrm{V}^{+}$ & $\mathrm{V}^{+}$ & $\mathrm{V}^{+}$ & $\mathrm{V}^{+}$ & $\mathrm{V}^{+}$ & V & $\mathrm{V}$ & V & $\mathrm{V}$ & $\mathrm{V}$ & $\mathrm{V}$ & $\mathrm{V}$ & $\mathrm{V}$ & & $\mathrm{V}$ & $\bar{V}$ \\
\hline Madrid (MAD) & CIEMAT, Casa de Campo $^{+}$ & $\mathrm{V}$ & $\mathrm{V}$ & $\mathrm{V}$ & $\mathrm{V}$ & $\mathrm{V}$ & $\mathrm{V}$ & $\mathrm{V}$ & $\mathrm{V}$ & $\mathrm{V}^{+}$ & $\mathrm{V}$ & $\mathrm{V}^{+}$ & $\mathrm{V}^{+}$ & & $\mathrm{V}$ & & \\
\hline Rome (ROM) & Buf $^{+}$ & $\mathrm{V}^{+}$ & $\mathrm{V}^{+}$ & $\mathrm{V}^{+}$ & $\mathrm{V}^{+}$ & $\mathrm{V}^{+}$ & $\mathrm{V}^{+}$ & $\mathrm{V}^{+}$ & $\mathrm{V}^{+}$ & & $\mathrm{V}^{+}$ & & & & & & \\
\hline Brisbane (BNE) & Rocklea $^{+}$ & $\mathrm{V}^{+}$ & $\mathrm{V}^{+}$ & $\mathrm{V}^{+}$ & $\mathrm{V}^{+}$ & $\mathrm{V}^{+}$ & $\mathrm{V}^{+}$ & $\mathrm{V}^{+}$ & $\mathrm{V}^{+}$ & & & $\mathrm{V}^{+}$ & $\mathrm{V}^{+}$ & & & $\mathrm{V}^{+}$ & \\
\hline Los Angeles (LA) & South Coast AQMD ${ }^{+}$ & $\mathrm{V}^{+}$ & $\mathrm{V}^{+}$ & $\mathrm{V}^{+}$ & $\mathrm{V}^{+}$ & & $\mathrm{V}^{+}$ & $\mathrm{V}^{+}$ & $\mathrm{V}^{+}$ & $\mathrm{V}^{+}$ & $\mathrm{V}^{+}$ & & $\mathrm{V}^{+}$ & & & V & \\
\hline
\end{tabular}


Table S4: Overall occurrence (\%) of each cluster at each site, classified into different categories (Traffic, Background, Nucleation and Specific case).

\begin{tabular}{|c|c|c|c|c|c|c|}
\hline Category & Subcategory & $\begin{array}{c}\text { Barcelon } \\
\mathrm{a}\end{array}$ & $\begin{array}{c}\text { Madri } \\
\mathrm{d}\end{array}$ & $\begin{array}{c}\text { Rom } \\
\mathrm{e}\end{array}$ & $\begin{array}{c}\text { Brisba } \\
\text { ne }\end{array}$ & $\begin{array}{c}\text { Los } \\
\text { Angeles }\end{array}$ \\
\hline \multirow{3}{*}{ Traffic } & Traffic 1 (T1) & $27 \%$ & $25 \%$ & $7 \%$ & $24 \%$ & $36 \%$ \\
\cline { 2 - 7 } & Traffic 2 (T2) & $24 \%$ & $22 \%$ & $27 \%$ & - & - \\
\cline { 2 - 7 } & Traffic 3 (T3) & $12 \%$ & $11 \%$ & $7 \%$ & $20 \%$ & $25 \%$ \\
\hline \multirow{4}{*}{$\begin{array}{c}\text { Backgroun } \\
\text { d }\end{array}$} & $\begin{array}{c}\text { Urban Background } \\
\text { (UB) }\end{array}$ & $15 \%$ & $6 \%$ & $25 \%$ & $22 \%$ & $6 \%$ \\
\cline { 2 - 7 } & $\begin{array}{c}\text { Summer } \\
\text { Background (SB) }\end{array}$ & - & $7 \%$ & - & - & - \\
\cline { 2 - 7 } & $\begin{array}{c}\text { Regional } \\
\text { Background (RB) }\end{array}$ & - & - & $28 \%$ & - & - \\
\hline Nucleation & Nucleation (NU) & $15 \%$ & $19 \%$ & $6 \%$ & $14 \%$ & $33 \%$ \\
\hline \multirow{2}{*}{$\begin{array}{c}\text { Specific } \\
\text { case }\end{array}$} & Nitrate (NIT) & $7 \%$ & $10 \%$ & - & - & - \\
\cline { 2 - 7 } & Growth 1 (G1) & - & - & - & $10 \%$ & - \\
\hline \multirow{2}{*}{ Total } & Growth 2 (G2) & - & - & - & $10 \%$ & - \\
\hline
\end{tabular}

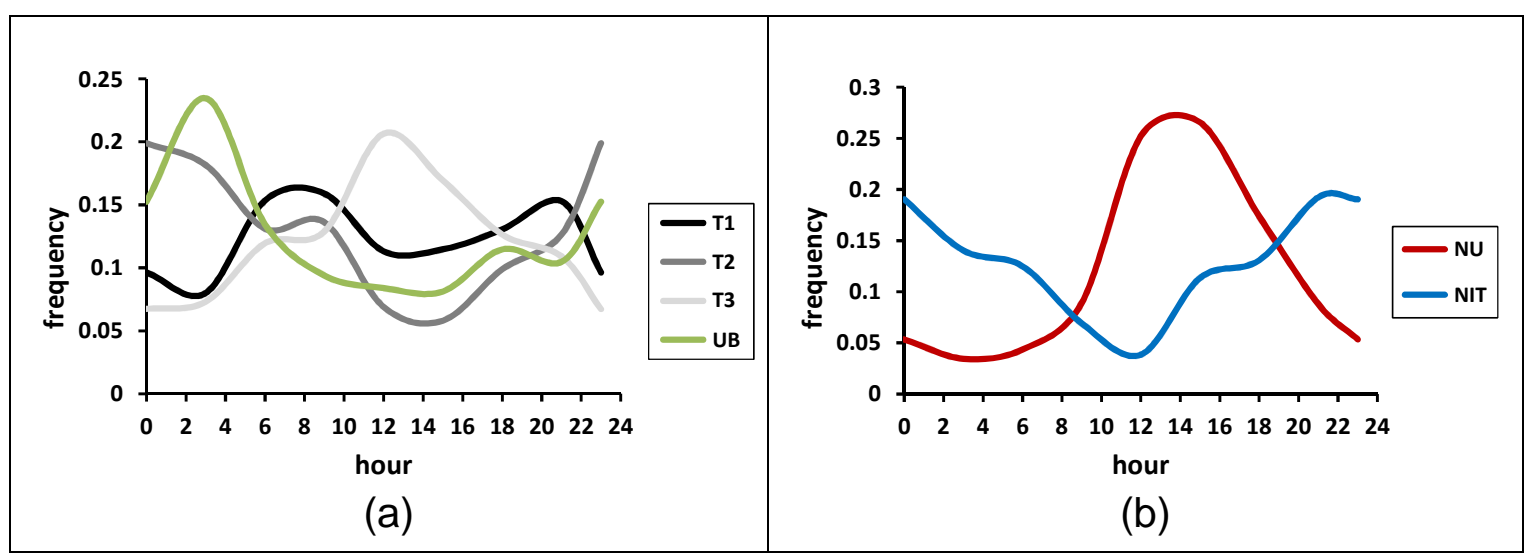

Figure S1: Diurnal trends for the main clusters: a) Traffic1 (T1), Traffic2 (T2), Traffic3 (T3) and Urban Background (UB); b) Nucleation (NU) and Nitrate (NIT). Although they are extracted from the results for Barcelona, they are representative of the rest of the cities. 


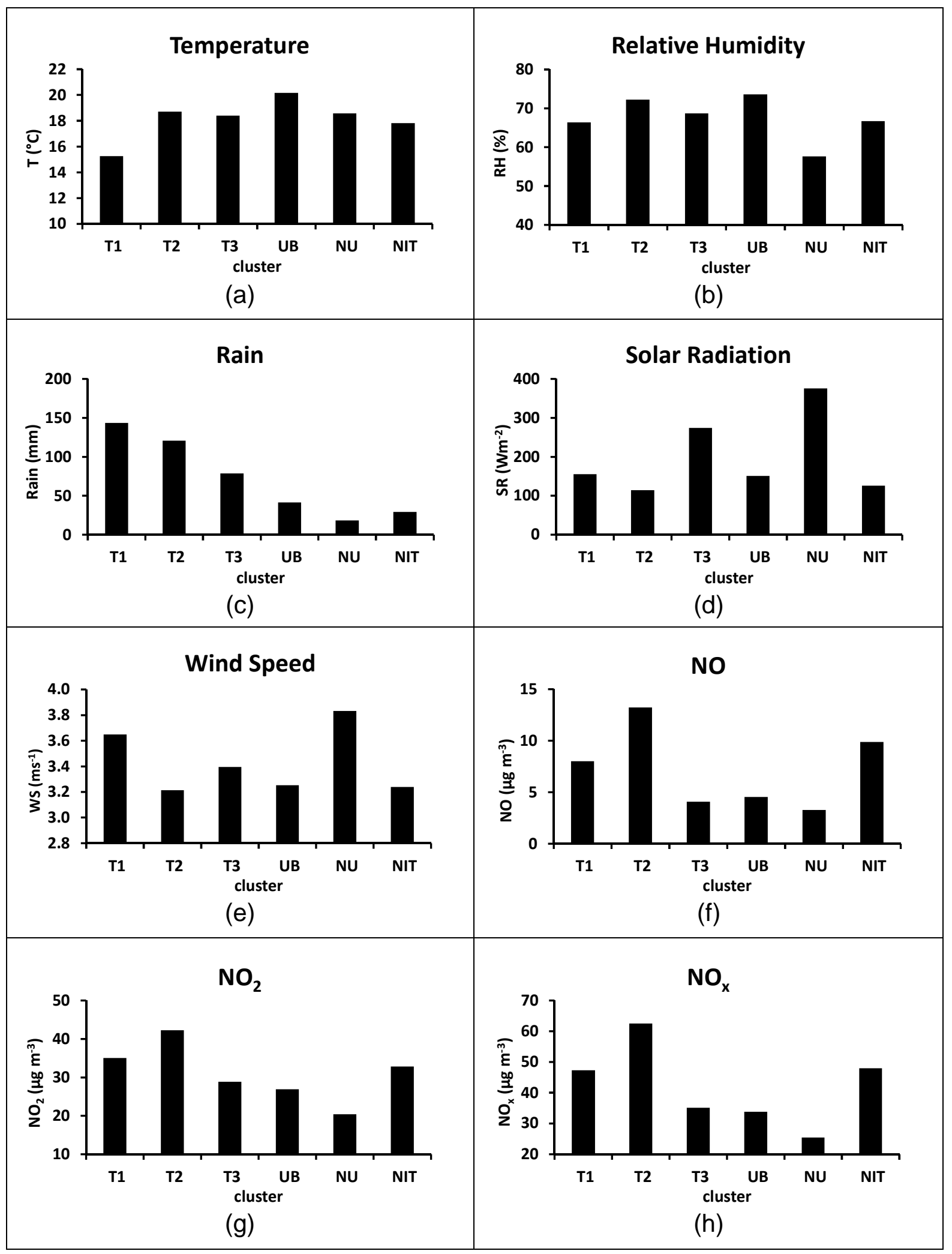




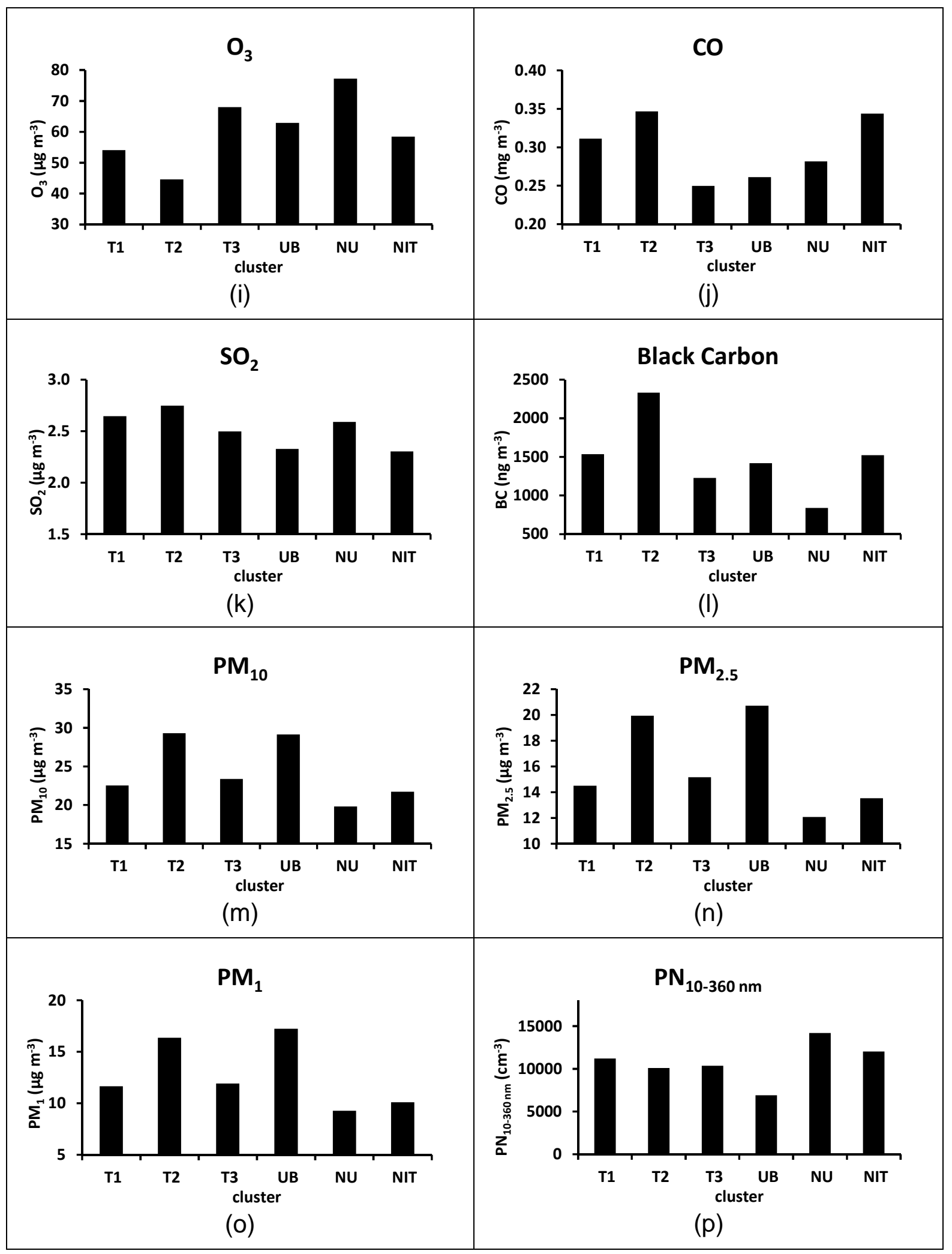

Figure S2: Meteorological parameters and gaseous pollutants for the main clusters: a) Temperature, b) Relative Humidity, c) Rain, d) Solar Radiation, e) Wind Speed, f) $\mathrm{NO}$, g) $\mathrm{NO}_{2}$, h) $\mathrm{NO}_{x}$, i) $\mathrm{O}_{3}$, j) $\mathrm{CO}, \mathrm{k}$ ) $\mathrm{SO}_{2}$, I) Black carbon, m) $\left.\mathrm{PM}_{10}, \mathrm{n}\right) \mathrm{PM}_{2.5}$, o) $\mathrm{PM}_{1}$, p) $\mathrm{PN}_{10-360}$. Although they are extracted from the results for Barcelona, the trend followed by clusters is representative of the rest of the cities. 


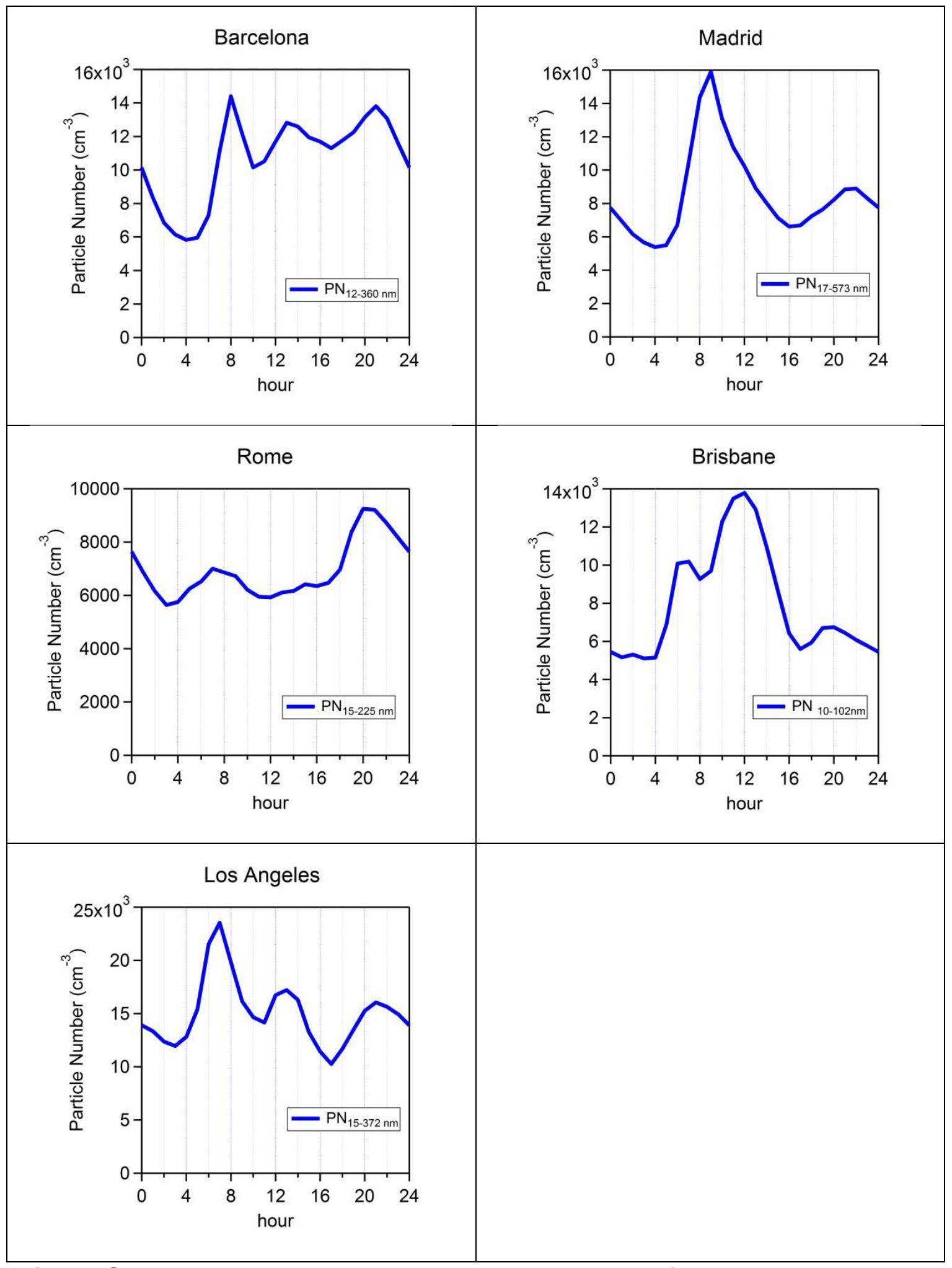

Figure S3: Average daily particle number concentration for the study periods at each city. 